\section{Spontaneous thrombosis of primary external jugular veins aneurysms}

\author{
Sergio Gianesini, Erica Menegatti, Michele \\ Zuolo, Savino Occhionorelli, Simona \\ Ascanelli, Paolo Zamboni
}

Vascular Disease Center, University of Ferrara, Italy

\section{Abstract}

External jugular vein (EJV) aneurysms represent a rare condition whose origin, evolution and consequent best therapeutic options still need further investigations. We present herein two peculiar clinical cases. In the first one, an EJV aneurysm developed around a malformed valve which embedded a spontaneous thrombosis. Transverse cutaneous nerve compression by the aneurysmatic mass was identified. In the second case, a recurring thrombosed EJV aneurysm was found pinched among the platysma muscle and the superficial layer of the cervical fascia. A pertinent literature review is also presented in order to interpret the findings herein never previously described.

\section{Introduction}

External jugular veins (EJV) aneurysms are isolated saccular or fusiform dilations that are rarely considered to lead to complications. ${ }^{1,2}$

Nevertheless, this general belief is biased by the rareness of the pathological condition, which hasn't allowed large case series studies so far. ${ }^{3}$ The EJV aneurysms are usually located at the supraclavicular region and its etiology remains uncertain among congenital (elastic tissue dysplasia) and acquired hypothesis (trauma, inflammation, thoracic outlet syndrome, neoplasms, parietal disease, venous hypertension) ${ }^{4,5}$

We herein present two peculiar EJV aneurysms clinical cases.

In the first one the aneurysm was topographically at the neck mid-portion and associated with a retroauricolar parestesia.

The second was responsible for a recurrent swimming-induced thrombosis.

A pertinent literature review is presented in ordered to clarify the pathogenetic mechanisms underlying the unusual pathological conditions described herein.

\section{Case Reports}

\section{Case 1: the higher than the usual} external jugular vein aneurysm with retroauricular parestesia

A 62-year-old female was evaluated for a swelling in the left neck mid-portion, which appeared 1 year prior and that had been slowly but progressively enlarging over time.

In the last month the bulging increased faster than before, becoming harder at the compression and associated with a retroauricular parestesia.

The patient's history reported just a goiter under medical control.

No previous trauma or chronic illness was reported. Blood tests and genetic tests for coagulation disorders were normal.

The physical examination revealed a $2.5 \mathrm{x} 3$ cm partially compressible non-tender, non-pulsative, fixed swelling, just above the sternocleidomastoid muscle and to the left of a mildly enlarged thyroid gland. No bruit was audible on ausculatation.

The bulging was not affected by the Valsalva manoeuver, but partially diminished from the standing to the supine position.

Chest and neck radiographs were normal.

Ultrasound investigation (ECD) was performed using MyLab ${ }^{\mathrm{TM}}$ (Esaote Group, Genoa, Italy) 70 (7.5-10 MHz linear array), setting the PRF (explain abbreviation) between 0.8 and $1.4 \mathrm{KHz}$.

ECD revealed a $2.7 \times 3.1 \mathrm{~cm}$ EJV aneurysm, partially compressible, filled in by an organized thrombus with initial recanalization signs.

The upper and lower EJV thirds were patent but extremely narrow, collapsing whenever moving the patient from the supine to the standing position, not-exhibiting an ECD-detectable flow, even at the lowest PRF scaninng.

The patient was operated under local anesthesia. A $2 \mathrm{~cm}$ long incision was performed on a skin crease at the neck mid-portion.

A partially thrombosed EJV aneurysm was found adherent to the transverse cutaneous branch of the great auricular nerve. An accurate dissection identified several tiny aneurysm collaterals, which were embedded in an inflammatory tissue (Figure 1).

The aneurysm was then ligated at both ends and excised.

Once the aneurysm was opened, a malformed monocuspid valve was identified in its lower third. The leaflet was enlarged, hypomobile and, surprisingly, reversed upside-down, with the cusp concavity facing the cranial side and filled in by the thrombus (Figure 2).

During the immediate post-operative time, the retro-auricular paresthesia mildly worsened, totally disappearing at the one month follow up. No others minor or major complica-
Correspondence: Sergio Gianesini, Vascular Disease Center, University of Ferrara, Via Aldo Moro 8, Cona, 44100 Ferrara, Italy. Tel. +39.0532.236524 - Fax: +39.0532.237144.

E-mail: sergiogianesini@hotmail.com

Key words: external jugular vein, aneurysm, thrombosis.

Contributions: SG, design, data acquisition, analysis and interpretation, manuscript drafting; EM, data acquisition, manuscript drafting; MZ, data acquisition; S0, SA, analysis and interpretation; PZ, design, analysis and interpretation, manuscript revision.

Conflict of interests: the authors declare no potential conflict of interests.

Acknowledgments: we thank Dr. Elly Wardle for her English revision of the manuscript.

Received for publication: 20 May 2013

Revision received: 17 June 2013.

Accepted for publication: 4 July 2013.

This work is licensed under a Creative Commons Attribution 3.0 License (by-nc 3.0).

(C) Copyright S. Gianesini et al., 2013

Licensee PAGEPress, Italy

Veins and Lymphatics 2013; 2:e17

doi:10.4081/vl.2013.e17

tions were reported.

Considering the malformed valve finding, at the 1 month follow up, a detailed ECD evaluation was precisely aimed to detect an eventually present chronic cerebro-spinal venous insufficiency, according to Zamboni's protocol: ${ }^{6}$ no pathological criteria were reported.

At 6 month follow up the patient was clinically and echographically in physiologic conditions.

\section{Case 2: the swimming-induced recurrent thrombosed external jugular vein aneurysm}

A 12-year-old female was evaluated for a tiny but bothering black dot on the neck skin. It suddenly appeared to the right of thyroid cartilage while the patient was swimming. The lesion was almost immediately followed by a craniallylocated EJV enlargement, with a consequent pain that forced the girl to get out from the water.

Patient's history, blood tests and genetic tests for coagulation disorders were unremarkable.

The physical examination identified a $2 \times 3$ $\mathrm{mm}$, not compressible, not movable, not pulsating, superficial, nodule-like structure, which was unaffected by active manoeuvres like Valsalva or straining. A mildly enlarged EJV was found running cranially to the mass. 
Interestingly, this vein was positive at the milking test, but with a delayed draining.

The ECD was performed using the same case 1 setting. It identified a mildly enlarged EJV (diameter, $2 \mathrm{~mm}$ ), showing a not ECD detectable at all the PRF values ranging from 0.8 to 1.4 assessments.

The vessel was compressible and ended in saccular thrombosed $1.8 \times 2.7 \mathrm{~mm}$ dilation on the superficial layer of the cervical fascia (Figure 3 ). The patient was treated for 1 month by means of low molecular weight heparin at therapeutic dosages.

At the end of the therapeutic time, the patient referred a progressive pain disappearance and no abnormal clinical signs were detected. The ECD assessed just a physiological $1 \mathrm{~mm}$ wide EJV.

Nevertheless, after another month, the clinical case recurred exactly in the same way, after the patient came back swimming.

Under local anesthesia a micro-incision was performed above the nodule-like mass. The surgical exploration revealed an EJV $1.5 \mathrm{x} 2 \mathrm{~mm}$ aneurysm that was entrapped among the superficial layer of the cervical fascia and the platysma muscle fibers. A delicate isolation led to the aneurysm excision after its both ends and collaterals ligation.

The aneurysmatic sac inner inspection identified the spherical-shaped thrombus.

Neither major nor minor complications were reported.

At 7 months follow up, the clinical case never recurred and the patient got back swimming.

\section{Discussion}

The EJV aneurysm is an usual pathological condition, whose etiopathogenesis and therapeutic approach still remain unclear. ${ }^{1,7}$

Moreover, just because of this condition rareness, both the clinical consequences and the potential complications of this condition needs further investigations. ${ }^{2,3}$

The first case presented herein offers several hints for a deeper analysis in future.

The EJV usually presents both a bicuspid valve at the subclavian confluence and in its middle portion. ${ }^{8}$

The higher than the usual EJV aneurysm localization of Case 1 coincides with the site of the reversed and malformed valve, found out on the surgical harvesting.

The internal and external jugular venous systems constitute collateral circulations with the thyroid veins and several others networks (such as lingual, facial, pharyngeal ones). ${ }^{9,10}$

We can assume that the patient's goiter could have constituted an extrinsic compression, thus representing an overload flow source for the jugular system.
It is reasonable that this overload then encountered a malformed valve as an obstacle to the drainage.

Consequently, this led to the increase in valve cusp transmural pressure, which is a well-known triggering factor for both aneurysmatic dilation and thrombotic complications. ${ }^{11}$

An obvious parallel is provided by the saphenous aneurysms, precisely occurring right after the subterminal valve. ${ }^{12}$

Certainly, it is not possible to discover if that leaflet enlargement and hypomobility is the cause or rather the consequence of the thrombotic pressure.

Nevertheless, the coincidental aneurysmatic localization in a reversed valve site paves the way for further investigations in the aneurysms origin.

Moreover, this clinical case points out a never-previously described cause of retroauricular paresthesia.

Along its course, the EJV crosses the transverse cutaneous branch of the great auricular nerve, running parallel to the latter in its upper third. ${ }^{13}$ It becomes evident how an inflammatory reaction could be triggered by an enlarging EJV aneurysm, pushing against the nerve, so resulting in a retro-auricular paresthesia.

The immediate post-operative paresthesia worsening could be linked to a dissectioninduced mild nerve shock, which spontaneously recovered over time. ${ }^{14}$

On the other hand, the second clinical case introduces the still unclear pathogenesis of cervical muscle entrapments and anomalies. ${ }^{15,16}$ Many variations have already been reported concerning the EJV, the great auricular nerve and the platysma muscle anatomy. ${ }^{17}$

The clinical case that was observed could be interpreted as an EJV nutcracker syndrome among a too narrow cervical fascia piercing and the platysma muscle. The head lateral turning during swimming represents a plausible triggering factor for the pathological onset.

Nevertheless, neither diagnostic nor histologic evaluations allowed us to exclude a congenital or primary venous disease.

Moreover, to our knowledge, this case depicts a never-previously reported recurrent EJV thrombosis.

Both the pathological scenarios described herein open up the discussion to what is to be considered as the best therapeutic approach.

Considering the rareness of the condition, up to now we are unaware of the real thrombotic and embolic risk linked to an EJV aneurysm.

In case of thrombosis, we have limited data to properly set our anti-thrombotic measurements. Are we going to consider it as a lower limb segmental superficial venous thrombosis and therefore not even administer heparin? Or are we ready to face the possible anti-thrombotic collateral effect to counteract a superfi-

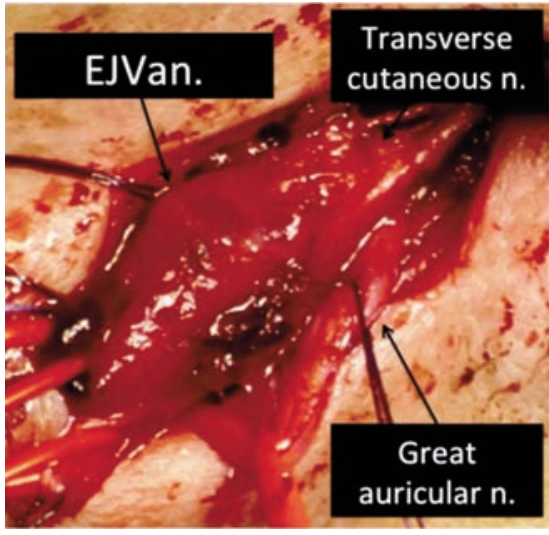

Figure 1. Intraoperative external jugular vein aneurysm (EJVan.) isolation, crossed by the transverse cutaneous nerve (n.). The great auricular nerve is isolated and gentlylaterally tractioned in order to avoid iatrogenic lesions. Many collaterals are embedded inside an inflammatory tissue

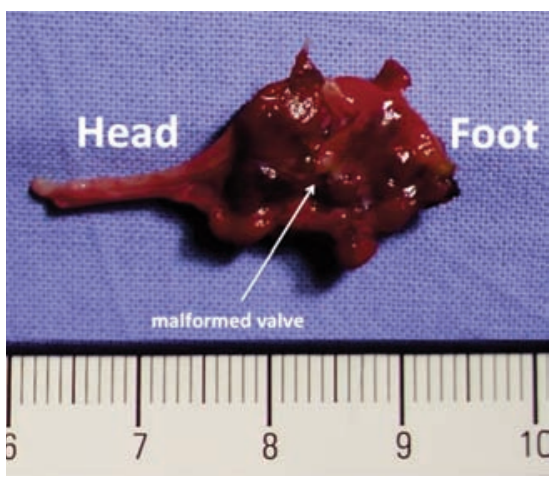

Figure 2. External jugular vein aneurysm post-operative opening. A reversed monocuspid fixed valve with a leaflet thickening is identified, together with the thrombus deposition above it.

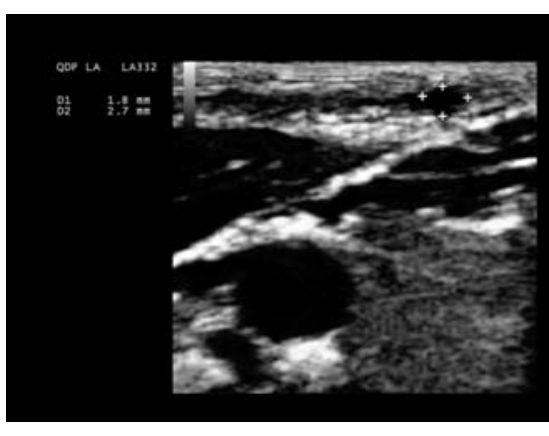

Figure 3. Ultrasound pre-operative scanning of a thrombosed $1.8 \times 2.7 \mathrm{~mm}$ external jugular vein aneurysm, among the platysma layer and the cervical fascia. 
cial venous thrombosis? ${ }^{18}$

The surgical approach has been considered relatively easily in the few previous reports. .,19,20 $^{2}$

Nevertheless, our data points out the importance of a delicate dissection because of the possible nervous structures anomalies and adherences to the same EJV: ${ }^{13,17}$ a potential cause of post-operative complications and legal consequences.

The same gold-standard treatment definition appears not to be so scientifically evident: what are the exact surgical indications?

The present work added some, yet definitely significant data to the amount of evidence that is still needed on this topic, paving the way for further investigations.

\section{Conclusions}

EJV aneurysms represent a rare condition, whose origin still needs further investigations.

According to our findings, a previously not so deeply evaluated, hemodynamic role seems to be mainly involved in the pathogenetic mechanism, leading to primary venous aneurysmatic dilations.

Both the prognosis and, in particular, the thrombo-embolic risk of this condition remain unclear. Wider case studies will be required to elucidate the best therapeutic approach.

\section{References}

1. Calligaro KD, Ahmad S, Dandora R, et al. Venous aneurysms: surgical indication and review of the literature. Surg
1995:117;1-6.

2. Karapolat S, Erkut B, Unlu Y. Multiple aneurysms of the left external jugular vein. Turk J Med Sci 2005;35:43-5.

3. Ioannou CV, Kostas T, Tsetis D, et al. External jugular vein aneurysm: a source of thrombotic complication. Int Ang 2010; 29:284-5.

4. Ekim H, Özen S. Primary venous aneurysm of the external jugular vein. Eur J Med 2002;7:24-5.

5. Battal B, Dursun E. External jugular vein aneurysm: clinical and radiologic imaging findings. Internet J Head Neck Surg 2009; 3:1a4e.

6. Nicolaides AN, Morovic S, Menegatti E, et al. Screening for chronic cerebrospinal venous insufficiency (CCSVI) using ultrasound: reccomendations for a protocol. Funct Neurol 2011;26:229-48.

7. Debaiyoti M, Bhupendra KJ, Pankaj KG, Anupama T. External jugular venous aneurysm: a clinical curiosity. J Nat Sci Biol Med 201;41:223-5.

8. Nisihara J, Takeuchi Y, Mijake M, Nagahata S. Distribution and morphology of valves in the human external jugular vein: indications for utilization in microvascular anastomosis. J Oral Maxillofac Surg 1996;54:879-82.

9. Zamboni P, Consorti G, Galeotti R, et al. Venous collateral circulation of the extracranial cerebrospinal outflow routes. Curr Neurovasc Res 2009;6:204-12.

10. Werner JD, Siskin GP, Mandato K, Englander M, Herr A. Review of venous anatomy for venographic interpretation in chronic cerebro-cpinal venous insufficiency. J Vasc Interv Radiol 2011;22:1681-90.

11. Karino T, Motomiya M. Flow through a venous valve and its implication for throm- bus formation. Thromb Res 1984;36:24557.

12. Pascarella L, Al-Tuwaijri M, Bergan JJ, et al. Lower extremity superficial venous aneurysms. Ann Vasc Surg 2005;19:69-73.

13. Lawrence E, Eicher G, Eicher SA. Great auricular nerve: anatomy and imaging in a case of perineural tumor spread. Am J Neuroradiol 2000;21:568-71.

14. Sharif-Alhoseini M, Rahimi-Movaghar V, Vaccaro AR. Underlying causes of paresthesia. In: Imbelloni LE, ed. Paresthesia. InTech; 2012. Available from: http://www. intechopen.com/books/paresthesia/underlying-causes-of-paresthesia

15. Takano T, Adachi H. On the anomalies in the infrahyoid muscles expecially in the omohyoid muscle. Acta Anat Nippon 1954; 29:5-6.

16. Gianesini S, Menegatti E, Mascoli F, et al. The omohyoid muscle entrapment of the internal jugular vein. A still unclear pathogenetic mechanism. Phlebology 2013. [In press].

17. Horacio J, Aboudib JH, Cardoso de Castro C. Anatomical variations analysis of the external jugular vein, great auricular nerve and posterosuperior border of the platysma muscle. Aesthet Plastic Surg 1997;21:75-8.

18. Colomina MJ, Godet C, Bagò J, et al. Isolated thrombosis of the external jugular vein. Surg Laparosc Endosc Pecutan Tech 2000;10:264-7.

19. Pandey M, Kumar P, Khanna AK. Phlebectasia of the external jugular vein. Int $\mathrm{J}$ Surg 2009;19.

20. Lee HY, Lee W, Cho YK, et al. Superficial venous aneurysm. L Ultrasound Med 2006; 25:771-6. 